\title{
A Discrete Duality Between Nonmonotonic Consequence Relations and Convex Geometries
}

\author{
Johannes Marti ${ }^{1} \cdot$ Riccardo Pinosio ${ }^{2}$
}

Received: 4 May 2017 / Accepted: 27 May 2019 / Published online: 10 June 2019

(C) The Author(s) 2019

\begin{abstract}
In this paper we present a duality between nonmonotonic consequence relations and well-founded convex geometries. On one side of the duality we consider nonmonotonic consequence relations satisfying the axioms of an infinitary variant of System $\mathrm{P}$, which is one of the most studied axiomatic systems for nonmonotonic reasoning, conditional logic and belief revision. On the other side of the duality we consider well-founded convex geometries, which are infinite convex geometries that generalize well-founded posets. Since there is a close correspondence between nonmonotonic consequence relations and path independent choice functions one can view our duality as an extension of an existing duality between path independent choice functions and convex geometries that has been developed independently by Koshevoy and by Johnson and Dean.
\end{abstract}

Keywords Convex geometries - Antimatroids · Nonmonotonic consequence relations · Conditional logic $\cdot$ Path independent choice functions $\cdot$ Duality

\section{Introduction}

In this paper we present a duality between the category of nonmonotonic consequence relations over complete atomic Boolean algebras and the category of well-founded convex geometries over the set of atoms of the algebra.

Nonmonotonic consequence relations are used in artificial intelligence as a framework for defeasible reasoning [24]. They also correspond to the nonnested fragment of a conditional logic developed in philosophy and linguistics [7, 25, 39]. However, the restriction to nonnested formulas is not essential for most properties of the logic. A third application of similar formal systems is in the theory of belief revision $[4,6,15]$. Tableaux and sequent

Johannes Marti

johannes.marti@gmail.com

Riccardo Pinosio

rpinosio@gmail.com

1 University of Strathclyde, Glasgow, UK

2 University of Amsterdam, Amsterdam, Netherlands 
proof systems for nonmonotonic consequence relations and conditional logics have been developed in [30, 33, 35].

The standard semantics for nonmonotonic consequence relations is given in terms of minimal elements in posets. If one considers the class of all posets then this semantics gives rise to an axiomatic system for nonmonotonic consequence relations that is called System $\mathrm{P}$. Completeness proofs for System $P$ with respect to the semantics on posets $[7,24,39]$ are technical and it is not obvious whether they lead to duality results. The reason for these difficulties is a mismatch between the semantics and the axioms of System P. It is noted in [39, sec. II.4.1] that the addition of a rather complex axiom, called coherence condition, greatly simplifies the completeness proof.

The comparatively high complexity of the coherence condition means that it is not expressible in the formal languages that are commonly used in the applications mentioned above. By increasing the expressivity of the language and assuming an analogue of the coherence condition [42] obtains a general representation result in relational structures that can be specialized to posets.

Our approach is to weaken the semantics instead of adding the coherence condition on the syntactic side. We use convex geometries, also known as antimatroids, as a semantics for nonmonotonic consequence relations satisfying the axioms of System P. This generalizes the poset semantics because for every poset one can define a convex geometry such that the extreme points of a set in the convex geometry are precisely the minimal elements of the set in the poset.

Convex geometries provide a combinatorial approach to the notion of convexity; for an overview see $[3,10,11]$ or $[21, \mathrm{ch}$. III]. The class of lattices arising as the lattice of convex sets in some convex geometry has been differently characterized by various authors; for a historical survey see [29].

Whereas the work mentioned in the previous paragraph is restricted to finite convex geometries there has recently been increased interest in the infinite case [1, 2, 41]. This work focuses on a specific class of infinite convex geometries which are algebraic closure operators, meaning that if a point is in the convex closure of a set it is already in the convex closure of one of its finite subsets. Algebraic convexities are of interest because the standard convexity of Euclidean spaces is algebraic. An equivalent condition is also assumed in the extensive study of convexity by [38] which however does not assume the anti-exchange axiom that is part of the definition of convex geometries.

For the duality of this paper we consider a different class of infinite convex geometries, which we call well-founded and which need not be algebraic. In a well-founded convex geometry every set has sufficiently many extreme points. This generalizes the notion of a well-founded poset, which are posets in which every set has sufficiently many minimal elements.

The idea of interpreting nonmonotonic consequence relations with respect to the family of convex sets in some convex geometry stands in the tradition of premise semantics, which interprets conditionals in arbitrary families of subsets of some set [23, 40]. A similar approach is developed in $[5,13,27]$. The contribution of this paper is to single out a class of families of subsets, namely the convex geometries, that gives rise to a duality with nonmonotonic consequence relations.

One can view our duality as an extension of the correspondence between path independent choice functions that are discussed in economics [32] and finite convex geometries. This correspondence has been developed independently by [22] and [18] and has been extended by [28] to a duality between semilattices of choice functions and semilattices of convex geometries. Moreover, [8] introduce the further concept of a framed dependence 
relation and prove that framed dependence relations correspond to path independent choice function and finite convex geometries. In [9] the correspondence between framed dependence relations and path independent choice functions is extended to the infinite case. It is also proven in [9] that the path independent choice function are precisely the unions of choice functions rationalized by well orders, which is a concept that is reminiscent of convex geometries. Since there is a close correspondence between path independent choice functions and nonmonotonic consequence relations our duality is an extension of these results. In particular, we characterize the infinite convex geometries that stand in the duality as the well-founded convex geometries and use morphisms that are structure preserving functions between the carriers of the nonmonotonic consequence relations or convex geometries, rather than only considering the inclusions between choice function or convex geometries over some fixed set as in [28].

The structure of this paper is as follows: In Section 2 we review nonmonotonic consequence relations in System P and derive some consequences of the axioms in System P. In Sections 3 and 4 we discuss how nonmonotonic consequence relations relate to path independence choice functions and to framed dependence relations. In Section 5 we review convex geometries. In Section 6 we introduce the notion of a smooth set which allows us to define well-founded convex geometries. We also prove a characterization of the smooth sets in terms of their extreme points. In Section 7 we prove the duality result of this paper.

\section{Nonmonotonic Consequence Relations}

In this section we introduce nonmonotonic consequence relations.

Definition 1 A nonmonotonic consequence relation for a set $W$ is a relation $\sim \subseteq \mathcal{P} W \times$ $\mathcal{P} W$ on the powerset $\mathcal{P} W$ of $W$ such that for all $A, B, C, D \in \mathcal{P} W$ and families $\mathcal{X} \subseteq \mathcal{P} W$ the following are satisfied:

- $\quad A \sim A$ (Id).

- If $A \sim C$ and $C \subseteq D$ then $A \sim D$ (RW).

- If $A \mid \sim X$ for every $X \in \mathcal{X}$ then $A \sim \sim \mathcal{X}\left(\right.$ And $\left.^{\infty}\right)$.

- If $A \sim B \cap C$ then $A \cap B \sim C$ (WCM).

- If $A \sim C$ and $B \mid \sim C$ then $A \cup B \sim C$ (Or).

A morphism from a nonmonotonic consequence relation $\sim^{\prime}$ for $W^{\prime}$ to a nonmonotonic consequence relation $\mid \sim$ for $W$ is a function $f: W \rightarrow W^{\prime}$ such that the inverse image map $f^{-1}[\cdot]: \mathcal{P} W^{\prime} \rightarrow \mathcal{P} W^{\prime}$ preserves the nonmonotonic consequence relation. This means that for all $A, C \in \mathcal{P} W^{\prime}$

$$
A \sim^{\prime} C \text { implies } f^{-1}[A] \sim f^{-1}[C] .
$$

A morphism $f$ of nonmonotonic consequence relations is strong if it reflects the nonmonotonic consequence relation. This means that the implication in Eq. 1 becomes a bi-implication.

One can think of a nonmonotonic consequence relation for $W$ as a relation on the Boolean algebra $\mathcal{P} W$. We are then restricting our attention to complete atomic Boolean algebras, which are precisely those Boolean algebras that can be represented as the powerset of their atoms [16, sec. 16]. Note that because every finite Boolean algebra is automatically complete and atomic our setting especially covers the case of all finite Boolean algebras. 
The definition of a morphism between nonmonotonic consequence relations is motivated by this representation of complete atomic Boolean algebras as powerset algebras. For every function $f: W \rightarrow W^{\prime}$ we have that $f^{-1}[\cdot]: \mathcal{P} W^{\prime} \rightarrow \mathcal{P} W$ is a complete Boolean algebra homomorphism between the powerset algebras and every complete Boolean algebra homomorphism arises in this way.

The axioms for nonmonotonic consequence relations in Definition 1 are an infinitary version of the rules in the well-known System P [24]. They are precisely the rules of System $P_{\infty}$ from [26] and differ from the rules of System $\mathrm{P}$ only in that in System $\mathrm{P}$ the infinitary $\left(\right.$ And $^{\infty}$ ) is replaced with its finitary variant (And), which holds for all $A, C, D \in \mathcal{P} W$ :

- If $A \mid \sim C$ and $A \sim D$ then $A \sim C \cap D$ (And).

Note that in the finite case the two axiomatizations coincide.

A minor difference between the axiomatization given here and the usual presentation of System $\mathrm{P}$ is that we found it more convenient to work with the weak cautious monotonicity rule (WCM) instead of the following more common cautious monotonicity rule (CM), which holds for all $A, B, C \in \mathcal{P} W$ :

- If $A \sim \sim B$ and $A \sim \sim$ then $A \cap B \sim \sim C(\mathrm{CM})$.

One can show that in the presence of (RW) and (And), where the latter is an obvious consequence of $\left(\mathrm{And}^{\infty}\right)$, the two rules are equivalent. To see this first assume that (WCM) holds. Then we can deduce (CM) because from the premises $A \mid \sim B$ and $A \mid \sim C$ it follows by (And) that $A \mid \sim B \cap C$, and hence we obtain $A \cap B \mid \sim C$ with (WCM). For the other direction assume that (CM) holds. Then we can deduce (WCM) because $A \mid \sim B \cap C$ entails by (RW) that both $A \mid \sim B$ and that $A \mid \sim C$, from which $A \cap B \mid \sim C$ follows by (CM).

In the following we make use of two consequences from the axioms in Definition 1, which we do not bother proving here in full detail.

The first is the following infinitary version of the (Or) rule, which holds for all $C \in \mathcal{P} W$ and families $\mathcal{X} \subseteq \mathcal{P} W$ :

- If $X \mid \sim C$ for every $X \in \mathcal{X}$ then $\bigcup \mathcal{X} \mid \sim C\left(\mathrm{Or}^{\infty}\right)$.

It follows from Example 7 together with Propositions 11 and 12 in [26] that $\left(\mathrm{Or}^{\infty}\right)$ is a consequence of the axioms in Definition 1.

The second is the following variant of the cautious cut rule, which holds for all $A, B, C \in$ $\mathcal{P} W$ :

- If $A \mid \sim B$ and $B \sim C$ then $A \cup B \sim C$ (CCut').

Let us see why this rule follows from another more common variant of the cautious cut rule, which in turn is shown in Lemma 5.3 of [24] and in Example 13 of [26] to follow from the axioms in Definition 1. This more common variant of the cut rule is the following rule (CCut): If $A \mid \sim B$ and $A \cap B \mid \sim C$ then $A \mid \sim C$. Thus assume (CCut) and that the premises $A \mid \sim B$ and $B \mid \sim C$ of (CCut') hold. From $A \mid \sim B$ it follows with an application of (Or) and (Id) that $A \cup B \mid \sim B$. Because $B=(A \cup B) \cap B$ we can write $B \mid \sim C$ as $(A \cup B) \cap B \sim C$. Now we can apply (CCut) with $A \cup B$ in the place of $A$ to obtain the needed $A \cup B \mid \sim C$.

Note that the derivation of (CCut) and hence of (CCut') from the rules in System $\mathrm{P}$ crucially uses classical complements in Boolean algebras. If one wishes to work with nonmonotonic consequence relations in a setting that is weaker than Boolean algebras it might be necessary to add (CCut) as an additional axiom. 


\section{Path Independent Choice Functions}

In this section we discuss the correspondence between nonmonotonic consequence relations and the path independent choice functions from [32]. This clarifies in what sense the results of this paper are an extensions of the duality developed by [18, 22, 28]. Readers who are only interested in the duality for nonmonotonic consequence relations can skip directely to Section 5 .

Choice functions are the same as selection functions, which have already been used in the semantics of conditional logic [31, 36, 39]. This semantics relies upon a correspondence analogous to that presented here. However in this context people have not considered path independence but rather used axioms that are direct translations of the axioms on nonmonotonic consequence relations from Definition 1.

Boolean algebras together with a selection function over the algebra are also the algebraic structures studied in the the duality of [42]. The axioms considered by [42] are close to those on selection functions mentioned in previous paragraph.

We now provide the definition of path independent choice functions.

Definition 2 A choice function for $W$ is a function $E: \mathcal{P} W \rightarrow \mathcal{P} W$ satisfying that $E(A) \subseteq$ $A$ for all $A \in \mathcal{P} W$.

A choice function $E: \mathcal{P} W \rightarrow \mathcal{P} W$ is path independent if for all $A, B \in W$ it holds that

$$
E(A \cup B)=E(E(A) \cup E(B)) .
$$

The correspondence between path independent choice functions and nonmonotonic consequence relations is given by the following two definitions.

Definition 3 For every nonmonotonic consequence relation $\mid \sim$ for $W$ define the induced function $E: \mathcal{P} W \rightarrow \mathcal{P} W$ such that for all $A \in \mathcal{P} W$

$$
E(A)=\bigcap\{C \in \mathcal{P} W|A| \sim C\} .
$$

Combining the previous definition with Definition 12 shows that via the duality of Section 7 the choice function $E$ maps a set in a convex geometry to the subset of its extreme points as in Definition 11.

Definition 4 For every choice function $E: \mathcal{P} W \rightarrow \mathcal{P} W$ for $W$ defined the induced relation $\sim$ for $W$ such that for all $A, C \in \mathcal{P} W$

$$
A \sim C \text { iff } E(A) \subseteq C
$$

It is easy to check that the previous two definitions establish a bijective correspondence between choice functions and relations satisfying (Id), (RW) and (And $\left.{ }^{\infty}\right)$. In the following two propositions we show that this correspondence restricts further to nonmonotonic consequence relations satisfying all the axioms of System $P_{\infty}$ and path independent choice functions.

Proposition 1 The choice function $E: \mathcal{P} W \rightarrow \mathcal{P} W$ induced by some nonmonotonic consequence relation $\sim \sim$ for $W$ is path independent. 
Proof Before we check path independence we make two observations. First note that $E(A) \cup E(B) \subseteq A \cup B$ for all $A, B \in \mathcal{P} W$. This holds because by (Id) we have $E(X) \subseteq X$ for every $X \in \mathcal{P} W$. The second observation is that for all $A, B \in \mathcal{P} W$ it holds that

$$
A \cup B \sim E(A) \cup E(B) .
$$

The claim (2) follows by (Or) because we have $A \sim \sim E(A) \cup E(B)$ and $B \sim E(A) \cup E(B)$, which can be obtained with (RW) from the fact that $X \sim E(X)$ for all $X \in \mathcal{P} W$. The latter holds because of $\left(\right.$ And $\left.^{\infty}\right)$.

Unfolding Definition 3 shows that to prove path independence of $E$ it suffices to show that for all $A, B, C \in \mathcal{P} W$

$$
A \cup B \mid \sim C \text { iff } \quad E(A) \cup E(B) \mid \sim C .
$$

For the left-to-right direction assume that $A \cup B \mid \sim C$. Since by Eq. 2 we also have that $A \cup B \mid \sim E(A) \cup E(B)$ it follows with $(\mathrm{CM})$ that $(A \cup B) \cap(E(A) \cup E(B)) \mid \sim C$. Since $E(A) \cup E(B) \subseteq A \cup B$ this is equivalent to $E(A) \cup E(B) \sim C$.

For the right-to-left direction assume that $E(A) \cup E(B) \mid \sim C$. Together with Eq. 2 we can then apply (CCut') to obtain $A \cup B \cup E(A) \cup E(B) \sim C$. Since $E(A) \cup E(B) \subseteq A \cup B$ this is equivalent to $A \cup B \sim C$.

Proposition 2 The relation $\mid \sim$ induced by some path independent choice function $E$ : $\mathcal{P} W \rightarrow \mathcal{P} W$ is a nonmonotonic consequence relation.

Proof We check that the relation induced by $E$ satisfies (WCM) and (Or).

To prove (WCM) we show that under the assumption that $E(A) \subseteq B$ it follows that $E(A \cap B)=E(A)$. This clearly entails (WCM) because if we are given $E(A) \subseteq B \cap C$ it follows that $E(A) \subseteq B$ and hence we obtain the required $E(A \cap B) \subseteq C$.

Hence assume $E(A) \subseteq B$. Because $E$ is a choice function we also have that $E(A) \subseteq A$ and hence it follows that $A \cap B=E(A) \cup(A \cap B)$. We then make the following computation

$$
\begin{aligned}
E(A \cap B) & =E(E(A) \cup(A \cap B))=E(E(E(A)) \cup E(A \cap B)) \\
& =E(E(A) \cup E(A \cap B))=E(A \cup(A \cap B))=E(A) .
\end{aligned}
$$

Here the second and fourth equality are applications of path independence. The third equality uses $E(E(A))=E(A)$, which is the trivial instance of path independence where the two sets are the same.

To prove (Or) first observe that for all $A, B \in \mathcal{P} W$ by path independence it holds that $E(A \cup B) \subseteq E(E(A) \cup E(B))$. Because $E$ is a choice function we have that $E(E(A) \cup$ $E(B)) \subseteq E(A) \cup E(B)$ and hence $E(A \cup B) \subseteq E(A) \cup E(B)$.

Now assume that the premises of (Or) are true, which means that $E(A) \subseteq C$ and $E(B) \subseteq$ $C$ for some $A, B, C \in \mathcal{P} W$. Then also $E(A) \cup E(B) \subseteq C$ and hence by the above we obtain the required $E(A \cup B) \subseteq C$.

One can show that morphisms between nonmonotonic consequence relations are precisely functions $f: W \rightarrow W^{\prime}$ that in terms of the corresponding choice functions satisfy the condition that $E\left(f^{-1}[A]\right) \subseteq f^{-1}\left[E^{\prime}(A)\right]$ for all $A \in \mathcal{P} W^{\prime}$. The strongness of a morphism $f: W \rightarrow W^{\prime}$ corresponds in terms of choice functions to the condition that $E^{\prime}(A) \subseteq f\left[E\left(f^{-1}[A]\right)\right]$ for all $A \in \mathcal{P} W^{\prime}$. In the context of choice or selection functions it might be more adequate to consider morphism that additionally satisfy the other inclusion, 
$E\left(f^{-1}[A]\right) \subseteq f^{-1}\left[E^{\prime}(A)\right]$ for all $A \in \mathcal{P} W^{\prime}$, of the condition on morphism. This notion of morphism is used in the setting of [42].

\section{Framed Dependence Relations}

In this section we discuss the connection between nonmonotonic consequence relations and the framed dependence relations from [8,9]. Similar relations have been studied in the context of conditional logic and nonmonotonic reasoning, where they are called relations of comparative possibility or relative likelihood. Examples of the former are in Section 2.5 of [25] or in [14] and examples of the latter are in Section 2.7 of [17] or in [37].

Definition 5 A dependence relation for a set $W$ is a relation $\succcurlyeq \subseteq \mathcal{P} W \times \mathcal{P} W$ on the powerset $\mathcal{P} W$ of $W$ such that for all $A, B, C \in \mathcal{P} W$ the following are satisfied:

- If $A \subseteq B$ then $B \succcurlyeq A$ (extension).

- If $A \succcurlyeq B$ and $B \succcurlyeq C$ then $A \succcurlyeq C$ (transitivity).

- If $A \succcurlyeq B$ and $A \succcurlyeq C$ then $A \succcurlyeq B \cup C$ (union).

A dependence relation $\succcurlyeq$ for $W$ is framed if the following holds for all $A \subseteq W$ and families $\mathcal{X} \subseteq \mathcal{P} W$ :

- $\quad$ If $X \subseteq A$ and $X \succcurlyeq A$ for every $X \in \mathcal{X}$ then $\bigcap \mathcal{X} \succcurlyeq A$.

The above definition of framed dependence relations is different, but easily seen to be equivalent to, the definition from [8], according to which a dependence relation $\succcurlyeq$ for $W$ is framed if for all $A \subseteq W$ there exists a least subset $A^{\prime} \subseteq A$ such that $A^{\prime} \succcurlyeq A$.

Definition 6 For every nonmonotonic consequence relation $\mid \sim$ for $W$ define the induced dependence relation $\succcurlyeq \subseteq \mathcal{P} W \times \mathcal{P} W$ such that for all $A, B \in \mathcal{P} W$

$$
A \succcurlyeq B \quad \text { iff } \quad B \cup A \mid \sim A \text {. }
$$

Proposition 3 The relation $\succcurlyeq \subseteq \mathcal{P} W \times \mathcal{P} W$ induced by a nonmonotonic consequence relation $\sim$ for $W$ according to Definition 6 is a framed dependence relation.

Proof (extension) follows immediately from (Id).

To see that $\succcurlyeq$ satisfies (transitivity) assume that $B \cup A \sim \sim A$ and $C \cup B \sim \sim B$. With (RW) it follows from the latter that $C \cup B \mid \sim B \cup A$. Applying (CCut) yields $C \cup B \cup A \mid \sim A$, which can be equivalently written as $C \cup B \cup A \mid \sim A \cap(C \cup A)$. We obtain the required $C \cup A \sim A$ with an application of (WCM).

(union) follows immediately from (Or).

That $\succcurlyeq$ is framed follows immediately from $\left(\mathrm{And}^{\infty}\right)$.

Definition 7 For every dependence relations $\succcurlyeq$ for $W$ define the induced nonmonotonic consequence relation $\sim \subseteq \mathcal{P} W \times \mathcal{P} W$ such that for all $A, B \in \mathcal{P} W$

$$
A \sim C \text { iff } A \cap C \succcurlyeq A
$$

Proposition 4 The relation $\sim \subseteq \mathcal{P} W \times \mathcal{P} W$ induced by a framed dependence relation $\succcurlyeq$ for $W$ according to Definition 7 is a nonmonotonic consequence relation. 
Proof (Id) follows immediately from (extension).

To see that $\sim \sim$ satisfies (RW) assume $A \cap C \succcurlyeq A$ and $C \subseteq D$. Hence $A \cap C \subseteq A \cap D$ and by (extension) we obtain $A \cap D \succcurlyeq A \cap C$. With (transitivity) can then conclude that $A \cap D \succcurlyeq A$.

(And ${ }^{\infty}$ ) follows immediately because $\succcurlyeq$ is framed.

To see that $\mid \sim$ satisfies (WCM) assume $A \cap B \cap C \succcurlyeq A$. Because $A \cap B \subseteq A$ we get $A \succcurlyeq A \cap B$ from (extension). Then $A \cap B \cap C \succcurlyeq A \cap B$ follows with (transitivity).

To see that $\sim \sim$ satisfies (Or) assume that $A \cap C \succcurlyeq A$ and $B \cap C \succcurlyeq B$. Using (extension) and (transitivity) we can derive that $(A \cup B) \cap C \succcurlyeq A$ and $(A \cup B) \cap C \succcurlyeq B$. With (union) we then obtain $(A \cup B) \cap C \succcurlyeq A \cup B$.

Proposition 5 Definitions 6 and 7 establish a bijective correspondence between nonmonotonic consequence relations and framed dependence relations over some set $W$.

Proof We show that the mappings from Definitions 6 and 7 are inverses to each other.

First consider a nonmonotonic consequence relation $\mid \sim$ on $W$, apply Definition 6 to obtain a framed dependence relation and the apply Definition 7 to obtain a nonmonotonic consequence relation $\mid \sim^{\prime}$. We need to prove that $A \mid \sim C$ iff $\left.A\right|^{\prime} C$ for all $A, C \subseteq W$. Unfolding the definition yields that $A \mid \sim^{\prime} C$ is equivalent to $A \mid \sim A \cap C$. Because of (RW) this entails $A \sim C$, and because of (Id) and (And) it is entailed by $A \sim C$.

Then consider a framed dependence relation $\succcurlyeq$ on $W$, apply Definition 7 to obtain a nonmonotonic consequence relation and the apply Definition 6 to obtain a framed dependence relation $\succcurlyeq^{\prime}$. We need to show that $A \succcurlyeq B$ iff $A \succcurlyeq B$. Unfolding the definition we see that the latter means that $A \succcurlyeq B \cup A$. This entails $A \succcurlyeq B$ because we can apply (transitivity) to the $B \cup A \succcurlyeq B$ we get with (extension). And, it is entailed by $A \succcurlyeq B$ because we can apply (union) to the $A \succcurlyeq A$ we get from (extension).

One might also define a notion of morphism between dependence relations. The most natural choice, analogous to the definition for nonmonotonic consequence relations, is to consider functions that preserve the dependence relation when taking preimages of sets under the function. Using the fact that unions and intersections are preserved under taking preimages it is then not difficult to show then that these morphism between framed dependence relations are then also precisely the morphism of nonmonotonic consequence relations between the nonmonotonic consequence relations corresponding to the frame dependence relations. Moreover, the morphism that reflect the framed dependence relation are precisely the strong morphism of the correspond consequence relations.

\section{Convex Geometries}

In this section we recall the notion of a convex geometry. Convex geometries can be equivalently defined either as some kind of closure operator, or as the lattice of closed sets of this closure operator, or as the lattice of complements of these closed sets, or as the interior operator of these complements.

A function $\mathrm{cl}: \mathcal{P} W \rightarrow \mathcal{P} W$ is a closure operator on a set $W$ if for all $X, Y \subseteq W$ it holds that $X \subseteq Y$ implies $\operatorname{cl}(X) \subseteq \operatorname{cl}(Y)$ (monotonicity), $X \subseteq \operatorname{cl}(X)$ (extensivity) and $\operatorname{cl}(X)=\operatorname{cl}(\operatorname{cl}(X))$ (idempotence). Note that in the presence of monotonicity and extensivity 
it always holds that $\operatorname{cl}(X) \subseteq \operatorname{cl}(\operatorname{cl}(X))$ and hence it suffices for idempotence to just proof $\operatorname{co}(\operatorname{co}(X)) \subseteq \operatorname{co}(X)$.

Given a closure operator $\mathrm{cl}: \mathcal{P} W \rightarrow \mathcal{P} W$ on $W$ a set $X \subseteq W$ is called closed if $X=$ $\operatorname{cl}(X)$. One can check that if $\mathcal{L} \subseteq W$ is a family of closed subsets of $W$ then its intersection $\bigcap \mathcal{L}$ is closed as well. Hence the closed sets of a closure operator form a complete meetsublattice of $\mathcal{P} W$. Conversely, given a complete meet-sublattice $\mathcal{L} \subseteq \mathcal{P} W$ one obtains a closure operator $\mathrm{cl}: \mathcal{P} W \rightarrow \mathcal{P} W$ on $W$ by letting $\mathrm{cl}(X)=\bigcap\{C \in \mathcal{L} \mid X \subseteq C\}$ for all $X \subseteq W$. It is straightforward to check that these simple constructions yield a one-to-one correspondence between closure operators on $W$ and complete meet-sublattices of $\mathcal{P} W$.

One might equivalently consider the complements of the closed sets in $\mathcal{L}$, which form a complete join-sublattice of $\mathcal{P} W$. Setting $\operatorname{int}(X)=\bigcup\left\{C^{c} \subseteq X \mid C \in \mathcal{L}\right\}$ for all $X \subseteq W$, where by $X^{c}=W \backslash X$ we denote the complement of $X$ relative to $W$, gives rise to an operator int : $\mathcal{P} W \rightarrow \mathcal{P} W$. This operator is an interior operator, meaning that it satisfies axioms that are dual to those on closure operators given above. Moreover, int, as defined above, is dual to $\mathrm{cl}$ in the sense that $(\operatorname{int}(X))^{c}=\operatorname{cl}\left(X^{c}\right)$ for all $X \subseteq W$.

Definition 8 A convex geometry on a set $W$ is a closure operator co : $\mathcal{P} W \rightarrow \mathcal{P} W$ over $W$ that satisfies the following anti-exchange property that for all $X \subseteq W$ and $u, v \in W$ with $u \neq v$ such that $u \notin \operatorname{co}(X)$ and $v \notin \operatorname{co}(X)$ it holds that $u \notin \operatorname{co}(X \cup\{v\})$ or $v \notin \operatorname{co}(X \cup\{u\})$.

The closure operator co of a convex geometry is also called convex closure. The family of closed subsets of a convex geometry is denoted by $\mathcal{C}$ and its members are called convex sets, while the family of complements of convex sets is denoted by $\mathcal{F}$ and its members are called feasible sets. We write fe $: \mathcal{P} W \rightarrow \mathcal{P} W$ for the interior operator given by the feasible sets. It is also called the feasible interior and satisfies that fe $(X)=\bigcup\{F \in \mathcal{F} \mid F \subseteq X\}=\operatorname{co}(X)^{c}$ for all $X \subseteq W$.

A morphism from a convex geometry co on $W$ to a convex geometry co' over $W^{\prime}$ is a function $f: W \rightarrow W^{\prime}$ such that for any $F$ that is feasible according to co it holds that its direct image $f[F]$ is feasible according to $\mathrm{co}^{\prime}$; in other words, morphisms between convex geometries preserve direct images of feasible sets. A morphism $f$ from co to co' is strong if for every feasible set $G$ in co' there is a feasible set $F$ in co such that $f[F]=G$; in other words, every feasible set is as the direct image of some feasible set.

The notion of morphism defined here is the same as the notion of a convex-to-convex function in [38, p. 15] and is closely related to the definition of a homomorphic image in [21, ch. III, sec 6]. More precisely, if one considers the convex geometry co' on $W^{\prime}$ that is the homomorphic image of a convex geometry co on $W$ under a function $f: W \rightarrow$ $W^{\prime}$ as defined in [21, ch. III, sec 6] then the function $f$ is a strong morphism from co to $\mathrm{co}^{\prime}$ in our terminology. However, our notion of morphism is unrelated to the notion of a convexity preserving function in [38, p. 15], which is analogous to that of continuous functions between topological spaces.

In what follows we switch freely between the different presentations of convex geometries as closure operators $\mathrm{co}, \mathrm{co}^{\prime}, \ldots$, families of closed sets $\mathcal{C}, \mathcal{C}^{\prime}, \ldots$, families of feasible sets $\mathcal{F}, \mathcal{F}^{\prime}, \ldots$ and interior operators fe, fe',$\ldots$.

Note that we do not require the empty set to be convex. This allows for a more elegant duality with nonmonotonic consequence relations.

It is possible to reformulate the anti-exchange in terms of convex and feasible sets. Let us say that a set separates two distinct points if it contains exactly one of them. Anti-exchange then requires that for every convex set and two distinct points outside of the set there is a convex set that extends the original set and separates the two points. For feasible sets it 
means that every feasible set which contains two distinct points contains another feasible set separating the points.

The notion of a convex geometry is a combinatorial abstraction of notions of convexity occurring in different contexts. The canonical example is the family of convex sets in a Euclidean space:

Example 1 Let $\mathbb{E}^{n}$ be the Euclidean space of dimension $n$. A subset of $\mathbb{E}^{n}$ is convex if for any two points in the subset every point on the straight line segment connecting the two points is in the subset. The family of convex sets so defined is closed under arbitrary intersections and satisfies the anti-exchange property, so it is a convex geometry.

Another example are the upset convexities of posets. These are relevant for this paper because they relate to the standard semantics for nonmonotonic consequence relations, which is defined on posets.

Example 2 Consider a poset $P=(W, \leq)$, that is a reflexive, transitive and anti-symmetric relation $\leq \subseteq W \times W$ on $W$. The upset closure of a set $X \subseteq W$ is its upset: $\uparrow X=\{w \in$ $W \mid w \geq x$ for some $x \in X\}$. Clearly, this satisfies the axioms for a closure operator and one can check that it also satisfies anti-exchange, so it is a convex geometry.

One can check that morphisms between the upset convexities of posets $(W, \leq)$ and $\left(W^{\prime}, \leq^{\prime}\right)$ are functions $f: W \rightarrow W^{\prime}$ which satisfy the back condition for bounded morphism for the relation given by the downwards directed order relation. This means that whenever $f(w) \geq v^{\prime}$ for some $w \in W$ and $v^{\prime} \in W^{\prime}$ then there is some $v \in W$ such that $w \geq v^{\prime}$ and $f(v)=v^{\prime}$. Strong morphisms are additionally surjective and order preserving, which means, that they are precisely the surjective bounded morphism.

Every finite convex geometry can be represented as the upset convexity in a poset up to a strong morphism of convex geometries $[12,20]$; for a clear exposition we refer to [21, ch. III, sec. 6]. This elegant representation, however, does not generalize to the infinite case. Infinite well-founded convex geometries, which are introduced later and are dual to nonmonotonic consequence relations, can still be represented by the upset convexity of a poset. We explain this in Section 8.

Further examples of convex geometries can be found in [21, ch. III, sec. 2].

\section{Extreme Points of Smooth Sets}

In this section we characterize the subsets in a convex geometry that are contained in the convex closure of their extreme points, which we call smooth. The well-founded convex geometries, which are part of our duality, are then defined to be those convex geometries in which all sets are smooth.

To define the notion of smooth set we need the notion of a trace [21, p. 27] or relative convexity [38, p. 13]. Traces are the obvious restrictions of a convex geometry on $W$ to a convex geometry on some subset $V \subseteq W$.

Definition 9 Fix a convex geometry $\mathcal{F}$ on $W$. For every set $V \subseteq W$ define the trace of $\mathcal{F}$ on $V$ to be the convex geometry $\mathcal{F}_{V}=\{F \cap V \subseteq V \mid F \in \mathcal{F}\}$ on $V$. 
It is it easy to see that the trace of a convex geometry $\mathcal{F}$ on a set $V \subseteq W$ is a convex geometry on $V$.

We could equivalently define traces in terms convex sets. A set $C^{\prime} \subseteq V$ is convex in the trace of $\mathcal{C}$ on $V$ iff $C^{\prime}=C \cap V$ for some $C \in \mathcal{C}$.

We are now ready to define the notions of a smooth set in a convex geometry and of a well-founded convex geometry. The notion of a smooth set is an adaptation of the notion of smooth set from Definition 3.8 in [24] to convex geometries. Our notion of a well-founded convex geometry is related to the version of the limit assumption that is formulated in [13, p. 215].

Definition 10 A convex geometry $\mathcal{F}$ on $W$ is smooth if for every feasible set $F \neq \emptyset$ there is a set $G$ that is a minimal element of the set $\{G \in \mathcal{F} \mid G \neq \emptyset, G \subseteq F\}$ for the ordering given by $\subseteq$.

A set $K$ in some convex geometry $\mathcal{F}$ is smooth if the trace of $\mathcal{F}$ on $K$ is smooth.

A convex geometry $\mathcal{F}$ on a set $W$ is well-founded if every subset $K \subseteq W$ is smooth in $\mathcal{F}$.

Note that every finite set in a convex geometry is smooth, hence every finite convex geometry is well-founded.

Remark 1 One has to be careful in formulating the definition of a smooth set $K \subseteq W$ directly in terms of the convex geometry $\mathcal{F}$ on $W$.

We first define for every $K \subseteq W$ the preorder $\subseteq_{K}$ on $\mathcal{P} W$ such that $X \subseteq_{K} \quad Y$ iff $K \cap X \subseteq K \cap Y$.

One can now check that a set $K \subseteq W$ is smooth iff for every feasible set $F$ with $K \cap F \neq$ $\emptyset$ there is a set $G$ that is a minimal element of the set $\left\{G \in \mathcal{F} \mid K \cap G \neq \emptyset, G \subseteq_{K} F\right\}$ for the ordering $\subseteq_{K}$.

In terms of convex sets a set $K \subseteq W$ is smooth iff for every convex set $C$ with $K \nsubseteq C$ there is a set $D$ that is a maximal element of the set $\left\{D \in \mathcal{C} \mid K \nsubseteq D, C \subseteq_{K} D\right\}$ for the ordering $\subseteq_{K}$.

The following example shows that it is too strong to reformulate smoothness of $K \subseteq W$ in a convex geometry $\mathcal{F}$ on $W$ to require that there exist suitable minimal elements with respect to the ordering $\subseteq$ on $W$. This justifies the restriction to the trace of $\mathcal{F}$ on $K$ and to the preorder $\subseteq_{K}$.

Example 3 Take the set $W=\omega+\{\star\}$ of natural numbers with one extra point added. Consider the convex geometry containing the following feasible sets:

$$
\mathcal{F}=\{F \subseteq W \mid \star \notin F\} \cup\{\uparrow n \cup\{\star\} \subseteq \omega \mid n \in \omega\}
$$

where $\uparrow n=\{m \in \omega \mid n \leq m\} . \mathcal{F}$ is closed under arbitrary unions and satisfies anti-exchange. Moreover one can show that it is well-founded. However there exists the infinite decreasing chain of feasible sets $\uparrow n \cup\{\star\}$ for each $n \in \omega$. Hence there is no minimal feasible set in the order $\subseteq$ that intersects with $\{\star\}$. Nevertheless $\{\star\}$ is smooth because relative to $\{\star\}$ the whole chain collapses to $\{\star\}$.

We now introduce the notion of the extreme points of a set in a convex geometry. 
Definition 11 Given a convex geometry co on $W$ we define for every $K \subseteq W$ the set $\operatorname{ex}(K) \subseteq W$ of extreme points of $K$ as

$$
\operatorname{ex}(K)=\bigcap\{X \subseteq W \mid K \subseteq \operatorname{co}(K \cap X)\} .
$$

For us it is most convenient to use the above definition of extreme points. In the literature one more commonly finds the definition that a point $w \in K$ is an extreme point of $K$ if $w \notin \operatorname{co}(K \backslash\{w\})$. It is not difficult to check that $\operatorname{ex}(K)$ as defined in Definition 11 is precisely the set of all extreme points in this sense.

Smooth sets are precisely those sets in a convex geometry for which the notion of extreme points as defined above is well-behaved. In particular, for a smooth set the set of its extreme points is the smallest subset such that its closure contains the original smooth set. This follows from the following theorem:

Theorem 1 The following are equivalent for every set $K \subseteq W$ in a convex geometry co on $W$ :

1. $K$ is smooth.

2. For every family $\mathcal{X} \subseteq \mathcal{P} W$ of subsets of $W$ it holds that whenever $K \subseteq \operatorname{co}(K \cap X)$ for all $X \in \mathcal{X}$ then also $K \subseteq \operatorname{co}(K \cap \bigcap \mathcal{X})$.

3. For all sets $X \subseteq W$ it holds that

$$
\operatorname{ex}(K) \subseteq X \quad \text { iff } \quad K \subseteq \operatorname{co}(K \cap X)
$$

\section{4. $K \subseteq \operatorname{co}(\operatorname{ex}(K))$}

Proof We start by showing that 1 implies 2 . Assume then that $K$ is smooth and take a family $\mathcal{X} \subseteq \mathcal{P} W$ such that $K \subseteq \operatorname{co}(K \cap X)$ for all $X \in \mathcal{X}$. We need to show that $K \subseteq$ $\operatorname{co}(K \cap \bigcap \mathcal{X})$.

First observe that we can assume without loss of generality that $\mathcal{X}$ is not empty, since if $\mathcal{X}$ is empty then the claim follows because by extensivity we have that $K \subseteq \operatorname{co}(K)=$ $\operatorname{co}(K \cap \bigcap \emptyset)$.

Assume then that $\mathcal{X}$ is not empty. Note that $K \subseteq \operatorname{co}(K \cap \bigcap \mathcal{X})$ is equivalent to the claim that $K \subseteq C$ for every convex set $C$ with $K \cap \bigcap \mathcal{X} \subseteq C$. Similarly, our assumption that $K \subseteq \operatorname{co}(K \cap X)$ for all $X \in \mathcal{X}$ is equivalent to the claim that $K \subseteq C$ for every convex set $C$ for which there is an $X \in \mathcal{X}$ with $K \cap X \subseteq C$.

Pick any convex set $C$ such that $K \cap \bigcap \mathcal{X} \subseteq C$. We want to show that $K \subseteq C$. Assume for a contradiction that $K \nsubseteq C$. From one of the reformulation of smoothness in Remark 1 it follows that there is a convex set $D$ with $C \subseteq_{K} D$ that is maximal in $\subseteq_{K}$ with the property that $D$ is convex, $C \subseteq_{K} D$ and $K \nsubseteq D$. It then follows that $K \cap X \nsubseteq D$ for all $X \in \mathcal{X}$, because otherwise from the assumption in the previous paragraph it would follow that $K \subseteq D$. This then means that for every $X \in \mathcal{X}$ there is some $p_{X} \in K \cap X$ such that $p_{X} \notin D$.

Since $\mathcal{X}$ is not empty there is some $X_{1} \in \mathcal{X}$. Set $p_{1}=p_{X_{1}}$ so that $p_{1} \in K \cap X_{1}$ and $p_{1} \notin D$.

We can then also show that there exists $p_{2} \neq p_{1}$ with $p_{2}=p_{X_{2}} \in K \cap X_{2}$ for some $X_{2} \in \mathcal{X}$ such that $p_{2} \notin D$. Otherwise, $p_{1}=p_{X} \in K \cap X$ for all $X \in \mathcal{X}$ and hence $p_{1} \in K \cap \bigcap \mathcal{X}$. This contradicts $p_{1} \notin D$ because we assumed that $K \cap \bigcap X \subseteq C$ hence $p_{1} \in K \cap C$; but we also had that $C \subseteq_{K} D$. 
Now we can apply anti-exchange, which yields a convex set $D^{+}$with $D \subseteq D^{+}$that separates $p_{1}$ and $p_{2}$. Assume without loss of generality that $p_{1} \in D^{+}$and $p_{2} \notin D^{+}$. From $p_{1} \in D^{+}$it follows that it is not the case that $D^{+} \subseteq_{K} D$ because $p_{1} \in K$ but $p_{1} \notin D$.

From $p_{2} \notin D^{+}$it follows that $K \nsubseteq D^{+}$because $p_{2} \in K$. We also have that $C \subseteq_{K} D^{+}$ because $C \subseteq_{K} D$. But now we can obtain a contradiction with the assumption that $D$ is maximal with respect to $\subseteq_{K}$ with the property that $D$ is convex, $C \subseteq_{K} D$ and $K \nsubseteq D$. Indeed, clearly $D^{+}$has this property and it is strictly above $D$ with respect to $\subseteq_{K}$ because $D \subseteq D^{+}$hence $D \subseteq_{K} D^{+}$and it is not the case that $D^{+} \subseteq_{K} D$.

Let us then consider the implication from 2 to 3 . First note that the direction from rightto-left in Eq. 3 is obvious from Definition 11. To show the direction from left-to-right assume that $\operatorname{ex}(K) \subseteq X$. To prove $K \subseteq \operatorname{co}(K \cap X)$ it then suffices by the monotonicity of co to show $K \subseteq \operatorname{co}(K \cap \operatorname{ex}(K))$. This however follows from claim 2 and the observation that $\operatorname{ex}(K)=\bigcap \mathcal{X}$, where $\mathcal{X}=\{X \subseteq W \mid K \subseteq \operatorname{co}(K \cap X)\}$.

Now consider the implication from 3 to 4 . First observe that by Definition 11 we have that $\operatorname{ex}(K) \subseteq K$ because by extensivity $K \subseteq \operatorname{co}(K \cap K)$. To show that $K \subseteq \operatorname{co}(\operatorname{ex}(K))$ apply the left-to-right direction of Eq. 3 to ex $(K) \subseteq \operatorname{ex}(K)$ yielding $K \subseteq \operatorname{co}(K \cap \operatorname{ex}(K))$. It follows that $K \subseteq \operatorname{co}(\operatorname{ex}(K))$ since $\operatorname{ex}(K) \subseteq K$.

Lastly we show the implication from 4 to 1 . So assume that $K \subseteq \operatorname{co}(\operatorname{ex}(K))$. We show that $K$ is smooth. By the first reformulation of smoothness in Remark 1 it suffices to show that for every feasible set $F$ such that $F \cap K \neq \emptyset$ there is a set $G$ that is minimal relative to the ordering $\subseteq_{K}$ for the property that $G$ is feasible, $K \cap G \neq \emptyset, G \subseteq_{K} F$.

Let then $F$ be such a feasible set. We first show that we can choose a $p \in \operatorname{ex}(K) \cap F$. Assume towards a contradiction that $\operatorname{ex}(K) \cap F=\emptyset$ and hence $\operatorname{ex}(K) \subseteq F^{c}$. From the assumption that $K \subseteq \operatorname{co}(\operatorname{ex}(K))$ it follows that $K \subseteq \operatorname{co}\left(F^{c}\right)$ since co is monotone. But since $F$ is feasible then $F^{c}$ is convex which means that $\operatorname{co}(F$ comple $)=F$ comple, hence $K \subseteq F$ comple. This contradicts the assumption that $K \cap F \neq \emptyset$.

Define now the set $G=(\operatorname{co}(K \backslash\{p\}))^{c}$. We now show that $K \cap G=\{p\}$. The inclusion $K \cap G \subseteq\{p\}$ follows because by the extensiveness of co we have that $K \backslash\{p\} \subseteq$ $\operatorname{co}(K \backslash\{p\})=$ Gcomple. For the other inclusion we need to show that $p \in K \cap G$. Clearly $p \in K$, so it remains to show that $p \in G$. Assume for a contradiction that $p \in$ Gcomple $=\operatorname{co}(K \backslash\{p\})$. By extensivity of co we have that $K \backslash\{p\} \subseteq \operatorname{co}(K \backslash\{p\})$, and hence $K \subseteq \operatorname{co}(K \backslash\{p\})=\operatorname{co}(K \cap(K \backslash\{p\}))$. By Definition 11 it follows that $\operatorname{ex}(K) \subseteq K \backslash\{p\}$, which contradicts the fact that $p \in \operatorname{ex}(K)$.

Using that $K \cap G=\{p\}$ we can show that $G$ has the minimality property stated above. Clearly $G$ is feasible since it is the complement of a convex set and $K \cap G \neq \emptyset$. Moreover, $G \subseteq_{K} F$ because $K \cap G=\{p\}$ and $p \in F$. Finally, $G$ is minimal with respect to $\subseteq_{K}$ for these properties because the only sets which are strictly below $G$ with respect to $\subseteq_{K}$ have an empty intersection with $K$.

Example 4 The Krein-Milman Theorem states that in $\mathbb{E}^{n}$ every compact convex set is the convex closure of its extreme points, which entails statement 4 of Theorem 1 . Hence all compact convex subsets of $\mathbb{E}^{n}$ are smooth in our terminology.

Example 5 Consider the case where co is the upset closure of a poset $P=(W, \leq)$. Then for any $K \subseteq W$ the set ex $(K)$ of its extreme points is precisely the set of the minimal elements of $K$ in the poset. As in [24], smoothness of a set $K$ means that for any element $w \in K$ there exists an element $v \in \operatorname{ex}(K)$ such that $v \leq w$. Well-foundedness of the upset closure co then amounts to well-foundedness of a poset in the usual sense. 
The standard semantics for nonmonotonic consequence relations on posets is defined such that $A \mid \sim C$ holds in some poset if and only if all minimal elements of $A$ are in $C$, where $A$ is assumed to be well-founded. Using (3) from Theorem 1 this semantic clause is equivalent to $A \subseteq \operatorname{co}(A \cap C)$. We use this formulation of the semantic clause for the duality result in the following section. An advantage of working with this reformulation of the semantic clause is that co is a monotone operator on $\mathcal{P} W$, whereas ex is not.

\section{The Duality}

In this section we prove the duality between nonmonotonic consequence relations and wellfounded convex geometries. We first show how to obtain a nonmonotonic consequence relation from a convex geometry, following the discussion in Example 5.

Definition 12 For every well-founded convex geometry co on $W$ we define the induced relation $\sim \subseteq \mathcal{P} W \times \mathcal{P} W$ for $W$ by setting for all $A, C \in \mathcal{P} W$

$$
A \sim C \text { iff } A \subseteq \operatorname{co}(A \cap C) .
$$

We show in Proposition 6 that the relation induced by a well-founded convex geometry is a nonmonotonic consequence relation.

In the other direction we can define an operator from a nonmonotonic consequence relation. Propositions 7, 8 and 9 below show that this definition yields a well-founded convex geometry.

Definition 13 For every nonmonotonic consequence relation $\mid \sim$ for $W$ we define the induced operator co : $\mathcal{P} W \rightarrow \mathcal{P} W$ on $W$ such that for all $C \subseteq W$

$$
\operatorname{co}(C)=\bigcup\{A \subseteq W|A| \sim C\}
$$

This definition of the induced operator is similar to the definition of the operator $\bar{C}$ from a choice function $C$ in [22, p. 37].

Proposition 6 The relation $\mid \sim$ induced by a well-founded convex geometry co is a nonmonotonic consequence relation for $W$.

Proof We have to check that the relation $\mid \sim$ from Definition 12 satisfies all the conditions on nonmonotonic consequence relations. This is easy to verify directly for all the conditions but $\left(\right.$ And $\left.^{\infty}\right)$. So we do those first.

For (Id) observe that $A \subseteq \operatorname{co}(A) \subseteq \operatorname{co}(A \cap A)$ because co is extensive.

For (RW) assume that $A \subseteq \operatorname{co}(A \cap C)$ and pick any $D$ such that $C \subseteq D$. We need that $A \subseteq \operatorname{co}(A \cap D)$, which holds because $A \cap C \subseteq A \cap D$ and co is monotone.

For (WCM) assume $A \subseteq \operatorname{co}(A \cap B \cap C)$. It then trivially follows that $A \cap B \subseteq$ $\operatorname{co}(A \cap B \cap C)$ because $A \cap B \subseteq A$.

For (Or) assume that $A \subseteq \operatorname{co}(A \cap C)$ and $B \subseteq \operatorname{co}(B \cap C)$. Because $A \cap C \subseteq(A \cup$ $B) \cap C$ it follows from the monotonicity of co that $A \subseteq \operatorname{co}((A \cup B) \cap C)$. Analogously we obtain $B \subseteq \operatorname{co}((A \cup B) \cap C)$ from $B \subseteq \operatorname{co}(B \cap C)$. Hence it follows that $A \cup B \subseteq$ $\operatorname{co}((A \cup B) \cap C)$. 
Lastly, consider $\left(\mathrm{And}^{\infty}\right)$. This follows directly from statement 2 in Theorem 1 . Note however that the application of this theorem presupposes that every subset of $W$ is smooth in co, which is just the well-foundedness of co. Moreover, note that the proof of Theorem 1 makes use of the exchange property.

Proposition 7 The operator co $: \mathcal{P} W \rightarrow \mathcal{P} W$ induced by a nonmonotonic consequence relation $\mid \sim$ for $W$ is a closure operator.

Proof (RW) implies that co is order preserving and (Id) implies that co is extensive.

To prove that co is idempotent first note that from applying $\left(\mathrm{Or}^{\infty}\right)$ to the union defining co in Definition 13 we obtain $\operatorname{co}(C) \mid \sim C$. We show that $\operatorname{co}(\operatorname{co}(C)) \subseteq \operatorname{co}(C)$. Since $\operatorname{co}(\operatorname{co}(C))$ is defined to be the union of all $A$ such that $A \mid \sim \operatorname{co}(C)$ it suffices to show that $A \subseteq \operatorname{co}(C)$ for every such $A$. Take any $A$ with $A \mid \sim \operatorname{co}(C)$. From (CCut') it follows that $A \cup \operatorname{co}(C) \mid \sim C$. Hence $A \cup \operatorname{co}(C) \subseteq \operatorname{co}(C)$, which clearly entails that $A \subseteq \operatorname{co}(C)$.

Proposition 8 The operator co $: \mathcal{P} W \rightarrow \mathcal{P} W$ induced by a nonmonotonic consequence relation $\sim$ for $W$ satisfies the anti-exchange property.

Proof We show that for all $X \subseteq W$ and $u, v \in W$ with $u \neq v$ if $u \in \operatorname{co}(X \cup\{v\})$ and $v \in \operatorname{co}(X \cup\{u\})$ then $u \in \operatorname{co}(X)$ and $v \in \operatorname{co}(X)$. This clearly suffices to obtain the contrapositive of anti-exchange.

From $u \in \operatorname{co}(X \cup\{v\})$ it follows that there is some $A$ such that $u \in A$ and $A \sim X \cup\{v\}$. Analogously it follows from $v \in \operatorname{co}(X \cup\{u\})$ that there is some $B$ such that $v \in B$ and $B \sim X \cup\{u\}$.

Consider $A \mid \sim X \cup\{v\}$. Because $u \in A$ this is equivalent to $A \cup\{u\} \mid \sim X \cup\{v\}$. Clearly this is equivalent to $A \cup\{u\} \mid \sim(X \cup\{u\} \cup\{v\}) \cap(X \cup\{v\})$. From (WCM) it follows that $(A \cup\{u\}) \cap(X \cup\{u\} \cup\{v\}) \sim X \cup\{v\}$, which is equivalent to $(A \cap(X \cup\{v\})) \cup\{u\} \mid \sim X \cup\{v\}$. If we then use (Or) with the instance $X \cup\{v\} \mid \sim X \cup\{v\}$ of (Id) we obtain $(A \cap(X \cup\{v\})) \cup$ $\{u\} \cup X \cup\{v\} \mid \sim X \cup\{v\}$. This simplifies to $X \cup\{u\} \cup\{v\} \sim X \cup\{v\}$.

Analogous reasoning applied to $B \sim X \cup\{u\}$ yields $X \cup\{u\} \cup\{v\} \mid \sim X \cup\{u\}$. But then we can use (And) to obtain $X \cup\{u\} \cup\{v\} \sim X$, which means that $u, v \in \operatorname{co}(X)$.

The previous propositions allow us to speak of the nonmonotonic consequence relation induced by a well-founded convex geometry and the convex geometry induced by a nonmonotonic consequence relation when referring to the constructions in Definitions 12 and 13.

Proposition 9 The convex geometry co on $W$ induced by a nonmonotonic consequence relation $\mid \sim$ for $W$ is well-founded.

Proof We show that every $K \subseteq W$ is smooth. Fix $K \subseteq W$. We use Theorem 1 and show that statement 2 is satisfied by $K$.

Consider any family $\mathcal{X} \subseteq \mathcal{P} W$ such that $K \subseteq \operatorname{co}(K \cap X)$ for every $X \in \mathcal{X}$. We need to show $K \subseteq \operatorname{co}(K \cap \cap \mathcal{X})$. We show that for every $X \in \mathcal{X}$ it holds that $K \mid \sim K \cap X$. From this it follows by $\left(\right.$ And $^{\infty}$ ) that $K \mid \sim K \cap \cap \mathcal{X}$ and hence we obtain $K \subseteq \operatorname{co}(K \cap \cap \mathcal{X}$ ) from Definition 13.

To see that $K \mid \sim K \cap X$ holds for every $X \in \mathcal{X}$ with $K \subseteq \operatorname{co}(K \cap X)$ fix an arbitrary such $X$. With (Id) and (RW) it follows that $K \mid \sim \operatorname{co}(K \cap X)$. Applying (Or $\left.{ }^{\infty}\right)$ to the union defining co in Definition 13 yields that $\operatorname{co}(K \cap X) \mid \sim K \cap X$. By (CCut') we obtain that $K \cup \operatorname{co}(K \cap X) \mid \sim K \cap X$. With (RW) we also get that $K \cup \operatorname{co}(K \cap X) \mid \sim K$ and hence it 
follows by $(\mathrm{CM})$ that $(K \cup \operatorname{co}(K \cap X)) \cap K \mid \sim K \cap X$. This is obviously equivalent to the required $K \sim K \cap X$.

We now show that these constructions behave well with respect to morphisms.

Proposition 10 Take a morphism of convex geometries $f: W \rightarrow W^{\prime}$ from a convex geometry co on $W$ to a convex geometry $\mathrm{co}^{\prime}$ on $W^{\prime}$. Then $f$ is a morphism of nonmonotonic consequence relations from the nonmonotonic consequence relation $\sim^{\prime}$ for $W^{\prime}$ induced by $\mathrm{co}^{\prime}$ to the nonmonotonic consequence relation $\sim \sim$ for $W$ induced by $\mathrm{co}$.

Proof To prove that $f$ is a morphism of nonmonotonic consequence relations we need to check that for all $A, C \subseteq W^{\prime}$ with $A \sim^{\prime} C$ it follows that $f^{-1}[A] \mid \sim f^{-1}[C]$. Take any such $A, C \subseteq W^{\prime}$ with $A \mid \sim^{\prime} C$. Because $\mid \sim^{\prime}$ is the nonmonotonic consequence relation induced by co' this means that $A \subseteq \operatorname{co}^{\prime}(A \cap C)$.

To prove $f^{-1}[A] \mid \sim f^{-1}[C]$ we show that $f^{-1}[A] \subseteq \operatorname{co}\left(f^{-1}[A] \cap f^{-1}[C]\right)$. Hence pick any $w \in f^{-1}[A]$. We derive a contradiction from the assumption that $w \notin$ $\operatorname{co}\left(f^{-1}[A] \cap f^{-1}[C]\right)$. It follows that

$$
w \in\left(\operatorname{co}\left(f^{-1}[A] \cap f^{-1}[C]\right)\right)^{c}=\mathrm{fe}\left(\left(f^{-1}[A] \cap f^{-1}[C]\right)^{c}\right)=\mathrm{fe}\left(f^{-1}\left[(A \cap C)^{c}\right]\right) .
$$

By the definition of fe then there is some feasible set $F \subseteq f^{-1}\left[(A \cap C)^{c}\right]$ such that $w \in F$. It follows that

$$
f(w) \in f[F] \subseteq f\left[f^{-1}\left[(A \cap C)^{c}\right]\right] \subseteq A \cap C^{c} .
$$

Because $f$ is assumed to be a morphism of convex geometries we have that $f[F]$ is a feasible set. Hence $f(w) \in \mathrm{fe}^{\prime}\left((A \cap C)^{c}\right)$ and so $f(w) \notin \mathrm{co}^{\prime}(A \cap C)$. From the assumption that $A \subseteq \operatorname{co}^{\prime}(A \cap C)$ it follows that $f(w) \notin A$ which is a contradiction to the assumption that $w \in f^{-1}[A]$.

Proposition 11 Take a morphism of nonmonotonic consequence relations $f: W \rightarrow W^{\prime}$ from a nonmonotonic consequence relation $\mid \sim^{\prime}$ for $W^{\prime}$ to a nonmonotonic consequence relation $\sim$ for $W$. Then $f$ is a morphism of convex geometries from the convex geometry co on $W$ induced by $\sim \sim$ to the convex geometry $\mathrm{co}^{\prime}$ on $W^{\prime}$ induced by $\sim^{\prime}$.

Proof To show that $f$ is a morphism of convex geometries we need to show that $f[F]$ is feasible whenever $F$ is. So pick any feasible $F \subseteq W$. We show that $f[F] \subseteq$ fe' $^{\prime}(f[F])$. Assume for a contradiction that we have a $v \in f[F]$ with $v \notin \mathrm{fe}^{\prime}(f[F])$. It follows that $v \in\left(\mathrm{fe}^{\prime}(f[F])\right)^{c}=\mathrm{co}^{\prime}\left((f[F])^{c}\right)$. Because $\mathrm{co}^{\prime}$ is induced by $\mid \sim^{\prime}$ this means that there is some $A \subseteq W^{\prime}$ with $A \sim^{\prime}(f[F])^{c}$ such that $v \in A$.

Because $f$ is a morphism of nonmonotonic consequence relations it follows that $f^{-1}[A] \mid \sim f^{-1}\left[(f[F])^{c}\right]$. Because $v \in f[F]$ there needs to be some $w \in F$ such that $f(w)=v$. It follows that $w \in f^{-1}[A]$ since we had that $v \in A$. Using the definition of co from $\mid \sim$ we obtain that $w \in \operatorname{co}\left(f^{-1}\left[(f[F])^{c}\right]\right)$. Using that $F \subseteq f^{-1}[f[F]]$ and the monotonicity of co we then have

$$
\operatorname{co}\left(f^{-1}\left[(f[F])^{c}\right]\right)=\operatorname{co}\left(\left(f^{-1}[f[F]]\right)^{c}\right) \subseteq \operatorname{co}\left(F^{c}\right)=F^{c} .
$$

Hence we can use $w \in \operatorname{co}\left(f^{-1}\left[(f[F])^{c}\right]\right)$ to obtain the contradiction that $w \notin F$.

We can also show that the property of being a strong morphism is preserved by our constructions. 
Proposition 12 Take a strong morphism of convex geometries $f: W \rightarrow W^{\prime}$ from a convex geometry co on $W$ to a convex geometry co' on $W^{\prime}$. Then $f$ is a strong morphism of nonmonotonic consequence relations from the nonmonotonic consequence relation $\mid \sim$ ' for $W^{\prime}$ induced by $\mathrm{co}^{\prime}$ to the nonmonotonic consequence relation $\mid \sim$ for $W$ induced by co.

Proof In order to show that $f$ is a strong morphism we show that the nonmonotonic consequence relation is reversed along $f^{-1}[\cdot]$. Hence, consider $A, C \subseteq W^{\prime}$ such that $f^{-1}[A] \mid \sim f^{-1}[C]$. This means that $f^{-1}[A] \subseteq \operatorname{co}\left(f^{-1}[A] \cap f^{-1}[C]\right)$. We need to show that $A \sim^{\prime} C$, that is, $A \subseteq \operatorname{co}^{\prime}(A \cap C)$.

We prove the contrapositive that for every $v \notin \operatorname{co}^{\prime}(A \cap C)$ it follows that $v \notin A$. From $v \notin \operatorname{co}^{\prime}(A \cap C)$ we obtain that $v \in \mathrm{fe}^{\prime}\left((A \cap C)^{c}\right)$ and hence there is some feasible set $G \subseteq(A \cap C)^{c}$ with $v \in G$.

Because $f$ is a strong morphism of convex geometries there is then a feasible set $F$ such that $f[F]=G$. This entails that $F \subseteq f^{-1}[f[F]]=f^{-1}[G]$. Moreover we have that $f^{-1}[G] \subseteq f^{-1}\left[(A \cap C)^{c}\right]=\left(f^{-1}[A \cap C]\right)^{c}=\left(f^{-1}[A] \cap f^{-1}[C]\right)^{c}$. Hence $F \subseteq$ $\left(f^{-1}[A] \cap f^{-1}[C]\right)^{c}$. Because $F$ is feasible it follows that $F \subseteq \mathrm{fe}\left(\left(f^{-1}[A] \cap f^{-1}[C]\right)^{c}\right)=$ $\left(\operatorname{co}\left(f^{-1}[A] \cap f^{-1}[B]\right)\right)^{c}$. Using the assumption that $f^{-1}[A] \subseteq \operatorname{co}\left(f^{-1}[A] \cap f^{-1}[C]\right)$ we get that $F \subseteq\left(f^{-1}[A]\right)^{c}=f^{-1}\left[A^{c}\right]$.

Now consider a $w \in F$ such that $f(w)=v$, which exists because $v \in G=f[F]$. From the last statement in the previous paragraph it follows that $w \in f^{-1}\left[A^{c}\right]$ which entails that $v \in A^{c}$ and we are done.

Proposition 13 Take a strong morphism of nonmonotonic consequence relations $f: W \rightarrow$ $W^{\prime}$ from a nonmonotonic consequence relation $\mid \sim^{\prime}$ for $W^{\prime}$ to a nonmonotonic consequence relation $\mid \sim$ for $W$. Then $f$ is a strong morphism of convex geometries from the convex geometry co on $W$ induced by $\mid \sim$ to the convex geometry co' on $W^{\prime}$ induced by $\sim^{\prime}$.

Proof Our assumption that $f: W \rightarrow W^{\prime}$ is a strong morphism of nonmonotonic consequence relations means that $f^{-1}[A] \sim f^{-1}[C]$ implies $A \sim^{\prime} C$ for all $A, C \subseteq W^{\prime}$. We need to show that $f$ is a strong morphism of convex geometries. To do so we show that for every feasible set $G$ in $\mathcal{F}^{\prime}$ it holds that $G=f\left[\mathrm{fe}\left(f^{-1}[G]\right)\right]$, and hence it arises as the image of a feasible set.

The right-to-left inclusion follows immediately because $f^{-1}[G] \supseteq \mathrm{fe}\left(f^{-1}[G]\right)$ and hence $G \supseteq f\left[f^{-1}[G]\right] \supseteq f\left[\mathrm{fe}\left(f^{-1}[G]\right)\right]$.

For the left-to-right inclusion we prove the contrapositive. Pick any $v \in W^{\prime}$ with $v \notin$ $f\left[\mathrm{fe}\left(f^{-1}[G]\right)\right]$. We want to show that then $v \notin G$.

First observe that $f^{-1}[\{v\}] \subseteq\left(\mathrm{fe}\left(f^{-1}[G]\right)\right)^{c}$, because otherwise there would be a $w \in$ $\mathrm{fe}\left(f^{-1}[G]\right)$ with $f(w)=v$ and hence we would have that $v \in f\left[\mathrm{fe}\left(f^{-1}[G]\right)\right]$, which yields a contradiction with our choice of $v$. It then follows that $f^{-1}[\{v\}] \subseteq \operatorname{co}\left(\left(f^{-1}[G]\right)^{c}\right)=$ $\operatorname{co}\left(f^{-1}\left[G^{c}\right]\right)$.

Now recall that by Definition $13 \operatorname{co}\left(f^{-1}\left[G^{c}\right]\right)=\bigcup\left\{B \subseteq W|B| \sim f^{-1}\left[G^{c}\right]\right\}$. Using the infinitary or rule (Or ${ }^{\infty}$ ) we obtain one single $B \subseteq W$ such that $f^{-1}[\{v\}] \subseteq B$ and $B \sim f^{-1}\left[G^{c}\right]$. Using (Id) and (Or) we obtain $B \cup f^{-1}\left[G^{c}\right] \mid \sim f^{-1}\left[G^{c}\right]$. Since $f^{-1}\left[G^{c}\right]=$ $\left(f^{-1}[\{v\}] \cup f^{-1}\left[G^{c}\right]\right) \cap f^{-1}\left[G^{c}\right]$ we can use (WCM) to conclude that $\left(B \cup f^{-1}\left[G^{c}\right]\right) \cap$ $\left(f^{-1}[\{v\}] \cup f^{-1}\left[G^{c}\right]\right) \mid \sim f^{-1}\left[G^{c}\right]$. By distributivity we get $\left(B \cap f^{-1}[\{v\}]\right) \cup f^{-1}\left[G^{c}\right] \mid \sim$ $f^{-1}\left[G^{c}\right]$ and because $f^{-1}[\{v\}] \subseteq B$ this is equivalent to $f^{-1}[\{v\}] \cup f^{-1}\left[G^{c}\right] \sim f^{-1}\left[G^{c}\right]$. Clearly this means that $f^{-1}\left[\{v\} \cup G^{c}\right] \mid \sim f^{-1}\left[G^{c}\right]$. 
From our assumption we now obtain that $\{v\} \cup G^{c} \mid \sim^{\prime} G^{c}$. By Definition 13 this entails $v \in \operatorname{co}^{\prime}\left(G^{c}\right)$. Since $G$ is feasible $G^{c}$ is convex and hence we have $\operatorname{co}^{\prime}\left(G^{c}\right)=G^{c}$. Therefore we obtain $v \in G^{c}$ which implies $v \notin G$ and we are done.

We can finally show that the correspondence between nonmonotonic consequence relations and convex geometries is bijective.

Proposition 14 Take a nonmonotonic consequence relation $\uparrow$ for $W$. Let co be the convex geometry on $W$ induced by $\sim$ and let $\sim$ ' be the nonmonotonic consequence relation for $W$ that is induced by co. It holds that $\sim^{\prime}$ is equal to $\sim$.

Proof We first show that $A \sim \sim C$ implies $A \mid \sim^{\prime} C$ for all $A, C \subseteq W$. Take $A, C \subseteq W$ with $A \mid \sim C$. We need to show that $A \mid \sim^{\prime} C$ which means that $A \subseteq \operatorname{co}(A \cap C)$. Using (Id) and (And) we obtain $A \sim A \cap C$ so $A \subseteq \bigcup\{B \subseteq W \mid B \sim A \cap C\}=\operatorname{co}(A \cap C)$.

To show that $A \mid \sim^{\prime} C$ implies $A \mid \sim C$ for all $A, C \subseteq W$ assume that $A \sim^{\prime} C$. We need to show that $A \mid \sim C$. The assumption that $A \mid \sim^{\prime} C$ means by definition that $A \subseteq \operatorname{co}(A \cap C)$. By (Id) and (RW) we first obtain that $A \mid \sim \operatorname{co}(A \cap C)$. From the definition $\operatorname{co}(A \cap C)=$ $\bigcup\{B \subseteq W|B| \sim A \cap C\}$ we can deduce with $\left(\mathrm{Or}^{\infty}\right)$ that $\operatorname{co}(A \cap C) \mid \sim A \cap C$. It follows using (CCut') that $A \cup \operatorname{co}(A \cap C) \sim A \cap C$. With (WCM) we obtain $(A \cup \operatorname{co}(A \cap C)) \cap A \mid \sim$ $C$, which is equivalent to $A \sim C$.

Proposition 15 Take a convex geometry co on $W$. Let $\sim$ be the nonmonotonic consequence relation for $W$ that is induced by $\mathrm{co}$ and let $\mathrm{co}^{\prime}$ be the convex geometry on $W$ that is induced by $\sim$. It holds that $\mathrm{co}^{\prime}$ is equal to $\mathrm{co}$.

Proof We first show that $\operatorname{co}(C) \subseteq \operatorname{co}^{\prime}(C)$ for all $C \subseteq W$. By definition $\operatorname{co}(C)=\bigcup\{A \subseteq$ $W|A| \sim C$ \} and hence it suffices to show that $\operatorname{co}(C) \mid \sim C$. By the definition of $\mid \sim$ this is equivalent to showing that $\operatorname{co}(C) \subseteq \operatorname{co}(\operatorname{co}(C) \cap C)$. But this is obvious since by the extensivity of co we have that $\operatorname{co}(C) \cap C=C$.

The other inclusion is that $\operatorname{co}^{\prime}(C) \subseteq \operatorname{co}(C)$. We can show this by picking any $A \subseteq W$ with $A \sim \sim C$ and showing that $A \subseteq \operatorname{co}(C)$. Fix such an $A$. By definition $A \sim \sim C$ entails that $A \subseteq \operatorname{co}(A \cap C)$ and hence $A \subseteq \operatorname{co}(C)$ because co is monotone.

We summarize the results from this section in the following theorem:

Theorem 2 The category of nonmonotonic consequence relations over complete atomic Boolean algebras satisfying the rules of System $P_{\infty}$ is dually equivalent to the category of well-founded convex geometries. The duality restricts to strong morphisms on both sides.

\section{Conclusion}

In this paper we have shown that nonmonotonic consequence relations over complete atomic Boolean algebras are dual to well-founded convex geometries. We hope that this result might lead to a transfer of insights between the study of nonmonotonic consequence relations and of convex geometries; two fields that, so far, have been developed independently of each other.

Our duality allows us to better understand the completeness proof for System $\mathrm{P}$ with respect to its order semantics [7, 24, 39], since it can be analyses as consisting of two steps. 
First we use the duality to construct a convex geometry from a nonmonotonic consequence relation. In the second step we represent this convex geometry as the upset convexity of a poset, up-to a strong morphism of convex geometries. In the finite case one can use the representation of $[12,20]$. We do not know of an analogous result in the literature that works also for infinite convex geometries. However, Theorem 4 in [9] and the completeness results for System $P_{\infty}$ in [26] suggest that such a representation might also be obtainable for well-founded infinite convex geometries.

A further question is whether System $\mathrm{P}$ is complete with respect to convexities that arise naturally in geometry. It is shown in [19], but see also [34] for a more accessible proof, that every finite convex geometry is isomorphic to the trace of a generalized convexity for $\mathbb{E}^{n}$ on finitely many points. The notion of a generalized convexity on $\mathbb{E}^{n}$ is introduced in this paper and distinct from the standard convexity on $\mathbb{E}^{n}$. Together with our duality this representation results immediately implies the weak completeness of System P with respect to such generalized convexities on $\mathbb{E}^{n}$. It is an open question whether this weak completeness can be adapted to the standard convexity on $\mathbb{E}^{n}$. For weak completeness it would suffice to represent an arbitrary finite convex geometry co by means of a strong morphism from the trace of the standard convexity on a finite subset of $\mathbb{E}^{n}$ to co, analogously to the representation of convex geometries by means of posets.

In [30] a labelled sequent calculus for System $\mathrm{P}$ is introduced in which points and sets of points are syntactic elements of the calculus. In light of our duality between System P and convex geometries it would be interesting to see whether this calculus could be reinterpreted in terms of points and convex sets. This might allow for the application of these proof systems to reason about convexity.

A further open problem is to characterize the morphism of choice functions $f: W \rightarrow W^{\prime}$ that satisfy $f^{-1}\left[E^{\prime}(A)\right] \subseteq E\left(f^{-1}[A]\right)$ for all $A \in \mathcal{P} W^{\prime}$ in terms of convex geometries. In the case of posets one can easily check, or adapt the results from [42], that these are the functions $f$ which are strictly order preserving, meaning that $w<v$ implies $f(w)<f(v)$ for all $w, v \in W$. We were unable to find a characterization for general well-founded convex geometries.

A final open question is whether the setting of this paper can be adapted to a duality between nonmonotonic consequence relations over arbitrary infinite Boolean algebras satisfying the axioms of System P and convex geometries over Stone spaces. This requires the formulation of suitable conditions ensuring the compatibility of the convexity with the Stone topology.

\section{Acknowledgments Research partially supported by EPSRC grant EP/N015843/1.}

Open Access This article is distributed under the terms of the Creative Commons Attribution 4.0 International License (http://creativecommons.org/licenses/by/4.0/), which permits unrestricted use, distribution, and reproduction in any medium, provided you give appropriate credit to the original author(s) and the source, provide a link to the Creative Commons license, and indicate if changes were made.

\section{References}

1. Adaricheva, K., Gorbunov, V., Tumanov, V.I.: Join-semidistributive lattices and convex geometries. Adv. Math. 173.1, 1-49 (2003)

2. Adaricheva, K., Nation, J.B.: A class of infinite convex geometries. Electron. J. Comb. 23, 1 (2016)

3. Adaricheva, K., Nation, J.B.: Convex geometries. In: Grätzer, G., Wehrung, F. (eds.) Lattice Theory: Special Topics and Applications, pp. 153-179. Springer (2016) 
4. Baltag, A., Smets, S.: Conditional doxastic models: A qualitative approach to dynamic belief revision. Electron. Notes Theor. Comput. Sci. 165, 5-21 (2006)

5. van Benthem, J., Pacuit, E.: Dynamic logics of evidence-based beliefs. Studia Logica 99.1-3, 61-92 (2011)

6. Board, O.: Dynamic interactive epistemology. Games Econ. Behav. 49.1, 49-80 (2004)

7. Burgess, J.: Quick completeness proofs for some logics of conditionals. Notre Dame J. Formal Logic 22.1, 76-84 (1981)

8. Danilov, V.I., Koshevoy, G.A.: A new characterization of the path independent choice functions. Math Soc. Sci. 51.2, 238-245 (2006)

9. Danilov, V.I., Koshevoy, G.A., Savaglio, E.: Hyper-relations, choice functions, and orderings of opportunity sets. Social Choice Welfare 45.1, 51-69 (2015)

10. Paul, H.: Edelman: Abstract convexity and meet-distributive lattices. In: Rival, I. (ed.) Combinatorics and Ordered Sets, vol. 57, pp. 127-150. Contemporary Mathematics (1986)

11. Edelman, P.H., Jamison, R.E.: The theory of convex geometries. Geometriae Dedicata 19.3, 247-270 (1985)

12. Edelman, P.H., Saks, M.E.: Combinatorial representation and convex dimension of convex geometries. Order 5.1, 23-32 (1988)

13. Girard, P.: From onions to broccoli: Generalizing Lewis' counterfactual logic. J. Appl. Non-Classical Logics 17.2, 213-229 (2007)

14. Girlando, M. et al.: Standard Sequent Calculi for Lewis' Logics of Counterfactuals. Logics in Artificial Intelligence. In: Michael, L., Kakas, A. (eds.), pp. 272-287. Springer (2016)

15. Grove, A.: Two modellings for theory change. J. Philos. Logic 17.2, 157-170 (1988)

16. Paul, R.: Halmos: Lectures on Boolean Algebras. Springer (1974)

17. Joseph, Y.: Halpern: Reasoning About Uncertainty. MIT Press (2003)

18. Johnson, M.R., Dean, R.A.: Locally complete path independent choice functions and their lattices. Math. Soc. Sci. 42.1, 53-87 (2001)

19. Kashiwabara, K., Nakamura, M., Okamoto, Y.: The affine representation theorem for abstract convex geometries. Comput. Geom. 30.2, 129-144 (2005)

20. Korte, B., Lovász, L.: Homomorphisms and Ramsey properties of antimatroids. Discret. Appl. Math. 15.2, 283-290 (1986)

21. Korte, B., Lovász, L., Schrader, R.: Greedoids. Springer (1991)

22. Koshevoy, G.A.: Choice functions and abstract convex geometries. Math. Soc. Sci. 38.1, 35-44 (1999)

23. Kratzer, A.: Partition and revision: The semantics of counterfactuals. J. Philos. Logic 10.2, 201-216 (1981)

24. Kraus, S., Lehmann, D., Magidor, M.: Nonmonotonic reasoning, preferential models and cumulative logics. Artif. Intell. 44.1-2, 167-207 (1990)

25. Lewis, D.: Counterfactuals. Blackwell (1973)

26. Marti, J., Pinosio, R.: A game semantics for system. Studia Logica 104.6, 1119-1144 (2016)

27. Marti, J., Pinosio, R.: Topological semantics for conditionals. In: Punčochár, V., Švarný, P. (eds.) The Logica Yearbook 2013. College Publications (2014)

28. Monjardet, B., Raderanirina, V.: The duality between the anti-exchange closure operators and the path independent choice operators on a finite Set. Math. Soc. Sci. 41.2, 131-150 (2001)

29. Monjardet, B.: A use for frequently rediscovering a concept. Order 1.4, 415-417 (1985)

30. Negri, S., Olivetti, N.: A sequent calculus for preferential conditional logic based on neighbourhood semantics. In: De Nivelle, H. (ed.) Automated Reasoning with Analytic Tableaux and Related Methods, pp. 115-134. Springer (2015)

31. Nute, D.: Topics in Conditional Logic. Reidel (1980)

32. Plott, C.R.: Path independence, rationality, and social choice. In: Econometrica, pp. 1075-1091 (1973)

33. Pozzato, G.L.: Conditional and preferential logics: Proof methods and theorem proving, vol. 208. Frontiers in Artificial Intelligence and Applications. IOS Press (2010)

34. Richter, M., Rogers, L.G.: Embedding convex geometries and a bound on convex dimension. Discret. Math. 340.5, 1059-1063 (2017)

35. Schröder, L., Pattinson, D., Hausmann, D.: Optimal tableaux for conditional logics with cautious monotonicity. In: Coelho, H., Studer, R., Wooldridge, M. (eds.) Proceedings ECAI 2010. Frontiers in Artificial Intelligence and Applications, vol. 215, pp. 707-712. IOS Press (2010)

36. Robert, C.: Stalnaker: A theory of conditionals. In: Rescher, N. (ed.) Studies in Logical Theory, pp. 98 112. Blackwell (1968)

37. Touazi, F., Cayrol, C., Dubois, D.: Possibilistic reasoning with partially ordered beliefs. J. Appl. Logic 13.4, 770-798 (2015) 
38. van de Vel, M.L.J.: Theory of Convex Structures. Elsevier (1993)

39. Veltman, F.: Logics for Conditionals. PhD thesis University of Amsterdam (1985)

40. Veltman, F.: Prejudices, presuppositions, and the theory of counterfactuals. In: Groenendijk, J., Stokhof, M. (eds.) Amsterdam Papers in Formal Grammar, vol. 1, pp. 248-282 (1976)

41. Wahl, N.: Antimatroids of finite character. J. Geom. 70.1, 168-175 (2001)

42. Wolter, F.: The algebraic face of minimality. Logic Logical Philos. 6.0, 225-240 (2004)

Publisher's Note Springer Nature remains neutral with regard to jurisdictional claims in published maps and institutional affiliations. 